\title{
Estudo da circulação e dos processos de mistura no extremo sul do mar de Cananéia: condições de dezembro de 1991
}

Luiz Bruner de MIRANDA; Afrânio Rubens de MESQUITA \& Carlos Augusto de Sampaio FRANÇA

\author{
Instituto Oceanográfico da Universidade de São Paulo
}

(Caixa Postal 66149, 05389-970 São Paulo, SP, Brasil)

- Abstract: Current and hydrographic properties sampled during three tidal cycles in December, 1991, were analysed. The highest values of the along channel velocity for the flood and ebb varied from $50 \mathrm{~cm} / \mathrm{s}$ to $-100 \mathrm{~cm} / \mathrm{s}$, respectively, and these values lead the high and low water occurrence with a time lag of 1.0-1.5 hours. The secondary circulation (cross channel component) predominates towards east with speeds in midwater depths up to $30 \mathrm{~cm} / \mathrm{s}$. Towards the west the secondary circulation was observed only in the upper layer with maxima speeds close to $-10 \mathrm{~cm} / \mathrm{s}$. Salinity variability indicates little vertical stratification and during the flood its highest value $(34.5-35.0 \%$ ) lags high waters at time intervals of 1.02.0 hours. However, during the ebb flow the low water leads the salinity minima $(29.5-30.5 \% 0)$ at time intervals between 2 and 3 hours. Although the studied region is part of a complex coastal plain estuary, the southern region of the Cananéia Sea was classified according to the Stratification-circulation Diagram, as partially mixed and weakly stratified (Type 2a). Its net motion reverses at $2.5 \mathrm{~m}$ depth and almost $100 \%$ of the upstream salt flux is due to turbulent diffusion.

- Resumo: Foram analisados dados de correntes e de propriedades hidrográficas amostradas durante três ciclos completos de maré, em dezembro de 1991. O máximo valor da componente longitudinal da velocidade da corrente variou, para as condições de enchente e de vazante, de 50 a $-100 \mathrm{~cm} / \mathrm{s}$ com esses extremos precedendo de 1,0 a 1,5 horas a ocorrência dos instantes de maré alta e baixa, respectivamente. A componente transversal de velocidade mostrou que a circulação secundária predominava na direção leste com núcleos de máxima de até $30 \mathrm{~cm} / \mathrm{s} \mathrm{em}$ profundidades médias, enquanto que no sentido oposto (para oeste) essa componente apresentou valores máximos próximos a $-10 \mathrm{~cm} / \mathrm{s}$ na camada de superfície. A variabilidade da salinidade indicou uma quase homogeneidade vertical e durante a enchente o seu máximo $(34,5-35,0 \%$ ) precede a maré alta com uma defasagem de 1,0-2,0 horas. Entretanto, durante a vazante, a maré baixa precede o mínimo de salinidade $(29,5-30,5 \% 0)$ com defasagens entre 2 e 3 horas. Não obstante a região estudada ser parte de um complexo sistema estuarino de planície costeira, o extremo sul do Mar de Cananéia foi classificado, de acordo com o Diagrama Estratificação-circulação, como parcialmente misturado e fracamente estratificado (Tipo 2a). O movimento resultante reverte a $2,5 \mathrm{~m}$ de profundidade e quase $100 \%$ do fluxo de sal estuário acima é devido a difusāo turbulenta.

- Descritors: Estuarine system, Classification, Hydrography, Circulation, Advection, Turbulent diffusion, Stratification- circulation Diagram.

- Descritores: Sistema estuarino, Classificação, Hidrografia, Circulação, Advecção, Difusāo turbulenta, Diagrama estratificação-circulação.

\section{Introdução}

O Mar de Cananéia, cuja parte sul é estudada neste trabalho, é um canal estuarino estreito e raso, forçado pela maré. É um dos principais canais do Sistema

Contr. $n^{\circ} 791$ do Inst. oceanogr. da Usp.
Estuarino-Lagunar de Cananéia- Iguape, localizado no extremo sul do Estado de São Paulo e delimitado pelas coordenadas Lat. $24^{\circ} 35^{\prime}$ S, Long. $047^{\circ} 30^{\prime} \mathrm{W}$ e Lat. $25^{\circ} 10^{\prime} \mathrm{S}$, Long. $048^{\circ} 05^{\prime} \mathrm{W}$. Esse sistema se compõe de um complexo sistema de canais entre três grandes ilhas (Cardoso, Cananéia e Comprida). A interligaçāo com a regiāo costeira adjacente mais próxima da regiāo investigada é através da Barra de Cananéia, embora mais 
Cananéia e Comprida). A interligação com a regiāo costeira adjacente mais próxima da regiāo investigada é através da Barra de Cananéia, embora mais remotamente as Barras de Icapara, ao norte, e de Ararapira, ao sul, contribuem para a troca de águas com a regiāo costeira adjacente (Fig. 1).
Desde 1950, quando da instalação da Base de Pesquisas do Instituto Oceanográfico da USP na Ilha de Cananéia, vários estudos oceanográficos foram realizados nessa regiāo. Besnard $(1950 a, b)$ fez uma descriçāo detalhada, apresentando informações sobre a sua gênese geológica. Machado (1952) realizou estudos físico-químicos preliminares das águas na junção do Canal

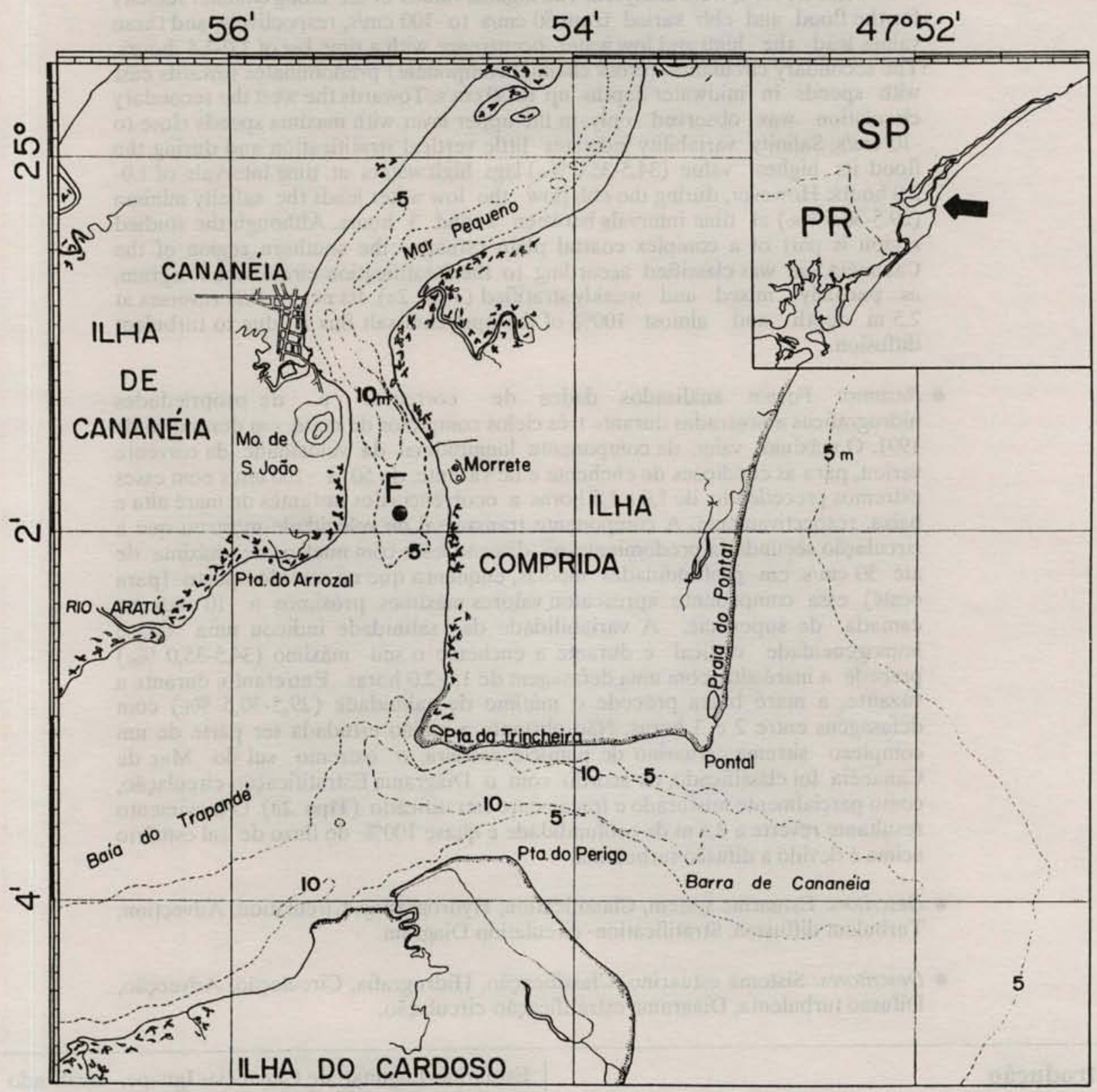

Fig. 1. Parte sul do sistema lagunar-estuarino Cananéia-Iguape e a localização da estação fixa $F$. 
de Ararapira e a Baía de Trapandé e Miniussi (1959) analisou a propagação da maré em torno da Ilha de Cananéia, identificando a região de encontro entre as ondas de maré que se propagam à partir das Barras de Cananéia e de Icapara.

Johannessen (1967) apresentou resultados da correlação entre parâmetros oceanográficos e meteorológicos e a variação do nível do mar; Tundisi (1969), em estudos sobre produção primária, analisou também variações de salinidade ocorridas em três estações fixas, amostradas no primeiro semestre de 1963, verificando nessa época que os extremos dessa propriedade ocorreram em fevereiro (mínimo) e maio (máximo).

Os trabalhos no campo da Oceanografia Física foram intensificados nos anos seguintes. Lorenzzetti (1976), analisando registros simultâneos de maré da Ilha do Bom Abrigo e de Cananéia, determinou a existência de um atraso de fase de aproximadamente 1 hora, com a maré oceânica liderando a maré de Cananéia. Miyao (1977) e Miyao et al. (1986) apresentaram resultados da distribuição de propriedades físicas e da circulação durante vários ciclos de maré no Mar de Cananéia no inverno e utilizando o critério de classificação, de acordo com a estratifição de salinidade, observaram que essa região comportava-se como do tipo parcialmente misturada. As constantes harmônicas de todas as constituintes da maré de Cananéia, bem como a sua variabilidade interanual no período de 1969-1974, foram calculadas por Mesquita \& Harari (1983). Nesse trabalho também podem ser obtidos dados estatísticos do nível médio do mar e sua variabilidade interanual, adequadamente relacionados a um nível de redução e ao zero do marégrafo. Com essas constantes harmônicas de Cananéia e Ubatuba, previsōes de maré para esses portos são regularmente publicadas desde 1988 (e.g. Mesquita \& Harari, 1993).

Analisando séries temporais de dados do Mar de Cananéia Miyao \& Harari (1989) apresentam um estudo preliminar da maré e das correntes de maré. Nesse estudo ficou demonstrado que a circulação nessa região é predominantemente gerada pela maré semidiurna, com valores extremos de $0,83 \mathrm{me} 0,13 \mathrm{~m}$ para as amplitudes de sizígia e quadratura, respectivamente. Embora os dados de corrente tenham sido obtidos a apenas $2 \mathrm{~m}$ do fundo ficou demonstrada uma assimetria nos valores extremos (de pico), com a velocidade de vazante maior do que a de enchente e o intervalo de tempo de enchente maior do que o de vazante.

Embora o sistema em estudo não tenha as características de um estuário clássico, o modelo analítico estacionário e bidimensional de Hansen \& Rattray (1966) foi aplicado por Miranda (1990) ao trecho sul do Mar de Cananéia, sob condições controladas das forçantes vento e descarga de água doce. Os perfis teóricos de velocidade de corrente calculados com esse modelo mostraram uma boa concordância com resultados experimentais nas condições de ausência de vento local e pequena descarga de água doce.

A água doce que chega ao sistema estuarino de Cananéia-Iguape é proveniente da drenagem continental de um grande número de pequenos rios (gambôas), cuja vazão não é monitorada. De acordo com Miyao et al. (1986), até o fechamento do Canal Valo Grande, em 1978, o Rio Ribeira de Iguape, com uma descarga parcial na parte norte do sistema através desse canal constituia, naquela época, a maior contribuição de água doce. Assim, a descarga de água doce nesse sistema, em volume por unidade de tempo, somente pode ser estimada com o conhecimento da área da bacia de drenagem e da diferença precipitação-evapotranspiração observada durante um determinado intervalo de tempo.

Estudos de climatologia do litoral sul do Estado de São Paulo, utilizando uma série temporal de dados meteorológicos da Estação Meteorológica da Base de Pesquisas do Instituto Oceanográfico da USP, foram apresentados por Occhipinti (1963). Dados mais recentes (Silva, 1989), indicam que a precipitação média mensal durante trinta anos (1956 a 1985) para o mês de dezembro é igual a $230 \mathrm{~mm}$. Entretanto, no mês de dezembro de 1991, época da realização do experimento, a precipitação mensal $(167,0 \mathrm{~mm})$ foi bem abaixo da média mensal histórica.

Os dados analisados neste trabalho foram obtidos num programa de medidas realizado no Mar de Cananéia, numa estação fixa (F) localizada nas proximidades de sua confluência com a Baía de Trapandé (Fig. 1), durante três ciclos completos de maré de sizígia (5-7 de dezembro de 1991) e constituem resultados parciais de um projeto mais amplo sob o tema Camada Limite de Fundo, financiado pela Fundação de Amparo à Pesquisa do Estado de São Paulo - FAPESP. Como resultado deste trabalho foi possível estudar a variação local de propriedades físicas e da circulação nessa região e também obter a sua classificação utilizando o Diagrama Estratificação-circulação, idealizado por Hansen \& Rattray (1966).

\section{Material e métodos}

Os dados hidrográficos e de correntes analisados neste trabalho foram obtidos na forma de séries temporais na estação fixa F (Fig. 1), durante três ciclos completos de maré no período de 5 a 7 de dezembro de 1991. Os perfilamentos das propriedades físicas e da velocidade da corrente foram feitos a intervalos de tempo de aproximadamente uma hora. Os trabalhos foram 
realizados a bordo do barco de pesquisas Albacora, do Instituto Oceanográfico da USP, que permaneceu fundeado com um sistema de âncoras de proa e de popa, para minimizar os efeitos da rotação da embarcação durante os sucessivos ciclos de maré.

A temperatura e a salinidade foram medidas ao longo da coluna de água em intervalos regulares de profundidade ( $\mathrm{z}$ ) e de tempo, com um Termosalinógrafo portátil, marca Beckman, modelo RS5-3, que se compōe de uma unidade submarina (célula indutiva) e de uma unidade de convés, onde sâo lidos os valores de temperatura em ${ }^{\circ} \mathrm{C}$ e de salinidade em \%o. Esse equipamento foi previamente calibrado no Laboratório de Instrumentação Oceanográfica (LIO) do Departamento de Oceanografia Física, esperando-se erros nāo superiores $\mathrm{a} \pm 0,1^{\circ} \mathrm{C}$ e $\pm 0,1 \%$ na determinação dessas variáveis independentes.

Os dados hidrográficos $(\mathrm{S}, \mathrm{T}, \mathrm{z})$ foram editados em formato adaptado do International Council for the Exploration of the Sea (ICES, 1966), para regiōes estuarinas de planície costeira, e processados para a determinação de propriedades físicas dependentes. Para a região em estudo a principal propriedade física dependente é a densidade, calculada pela Equação Internacional de Estado da Água do Mar (EIE-1980). Como é usual para estuários de planície costeira, neste trabalho a densidade está expressa pela "anomalia da densidade à pressão atmosférica" $\left(\sigma_{t}\right)$ que foi calculada em função da densidade, pela seguinte equação:

$$
\sigma_{\mathrm{t}}=\rho(\mathrm{S}, \mathrm{T}, 0)-1000, \mathrm{~kg} / \mathrm{m}^{3}
$$

onde $\rho(\mathrm{S}, \mathrm{T}, 0)$ indica a densidade à pressāo atmosférica, em unidades $\mathrm{de} \mathrm{kg} / \mathrm{m}^{3}$.

A velocidade da corrente (intensidade e direção) foi medida com um correntômetro fabricado pela CONSUB, modelo COR-04/500. Essas medidas foram feitas a intervalos regulares de profundidade $(\delta \mathrm{z}=1 \mathrm{~m})$ até uma distância aproximada do fundo de $0,60 \mathrm{~m}$. A intensidade desse equipamento foi calibrada no tanque de provas do Instituto de Pesquisas Tecnológicas (IPT), obtendo-se para o sensor de velocidade a seguinte equação de calibraçāo que permite o cálculo da intensidade corrigida, V:

$$
\mathrm{V}=8,96+1,16 \mathrm{~V}_{\mathrm{i}}
$$

onde $V_{i}$ é a intensidade medida pelo instrumento. Essa equação indica que o correntômetro utilizado é pouco sensível a baixas velocidades e que o mesmo não é capaz de medir velocidades menores do que $9,0 \mathrm{~cm} / \mathrm{s}$. O valor corrigido da intensidade apresenta raiz do desvio médio quadrático igual a $\pm 4,1 \mathrm{~cm} / \mathrm{s}$, valor este muito próximo ao erro indicado no manual do instrumento $( \pm$ $5,0 \mathrm{~cm} / \mathrm{s})$. A incerteza nas medidas da direção, de acordo com esse manual, é igual a $\pm 5^{\circ}$.

Para um estudo das características hidrodinâmicas da região em estudo, todos os valores do vetor velocidade da corrente foram decompostos nas componentes longitudinal (v) e transversal ou secundária $(u)$ ao canal, segundo o seguinte sistema de coordenadas: $\mathrm{O}_{\mathrm{y}}$ orientado ao longo do canal, positivo no sentido norte, $\mathrm{O}_{\mathrm{x}}$ orientado para a direita (da Ilha de Cananéia para a Ilha Comprida); para essa decomposiçâo foi levada em conta a declinação magnética local $\left(18,4^{\circ} \mathrm{W}\right)$. O eixo da profundidade $\left(\mathrm{O}_{\mathrm{z}}\right)$ foi orientado contrário à aceleração da gravidade.

Para minimizar os efeitos das variações da profundidade da coluna de água devido à oscilação da maré, a profundidade $z$ de amostragem foi transformada na profundidade adimensional $(\mathrm{Z})$, definida por $\mathrm{Z}=\mathrm{z} / \mathrm{L}$, onde $\mathrm{L}$ indica a profundidade local no instante da amostragem, de acordo com a metodologia estabelecida por Kjerfve (1975). Logo, $Z=0$ e $Z=1$ correspondem à superfície $(z=0)$ e ao fundo $(z=L)$, respectivamente, e todos os dados experimentais amostrados a uma profundidade $\mathrm{z}$ foram interpolados a intervalos constantes da profundidade $\mathrm{Z}(\Delta \mathrm{Z}=\mathrm{Z} / 10)$.

Com a introduçāo desse sistema de referência $\left(\mathrm{O}_{\mathrm{xyz}}\right)$, segue-se que o campo das propriedades físicas $\mathrm{S}, \mathrm{T}$ e $\sigma_{\mathrm{t}}$, e as componentes $\mathrm{u}$ e $\mathbf{v}$ de velocidade amostrada na estação fixa representam relaçōes funcionais do tipo $\mathrm{S}=\mathrm{S}(\mathrm{Z}, \mathrm{t}), \mathrm{T}=\mathrm{T}(\mathrm{Z}, \mathrm{t}), \sigma_{\mathrm{t}}=\sigma_{\mathrm{t}}(\mathrm{Z}, \mathrm{t}), \mathrm{u}=\mathrm{u}(\mathrm{Z}, \mathrm{t}) \mathrm{e} \mathrm{v}$ $=\mathrm{v}(\mathrm{Z}, \mathrm{t})$, respectivamente. Para a análise da distribuição dessas quantidades em funçāo do espaço (profundidade adimensional) e do tempo (horas), referidas ao meridiano de Greenwich (GMT), essas relações funcionais foram representadas graficamente pelo método das isopletas.

Os valores horários das propriedades físicas, obtidos na estação fixa (F), foram utilizados para calcular os perfis médios (média no tempo) e os valores médios (médias no tempo e no espaço) da salinidade e da componente longitudinal da velocidade da corrente (corrente residual). Detalhes da metodologia utilizada para a determinação desses valores médios podem ser obtidos em Miranda (1984).

O sistema estuarino em estudo foi classificado qualitativamente à partir da estratificação de salinidade, de acordo com Pritchard (1952) e Officer (1977). Para uma classificação quantitativa e uma análise da importância relativa dos processos advectivo e da difusão turbulenta, foi utilizado o Diagrama Estratificaçāo-circulaçāo, idealizado por Hansen e Rattray (1966), com base num modelo bidimensional, estacionário, lateralmente homogêneo e com geometria simples (seção retangular). Nesse diagrama, a ordenada e 
abscissa representam os parâmetros estratificação e circulação, respectivamente, definidos por:

$$
\delta S_{/} S_{0} \text { e } V_{s} / V_{f} \text {, }
$$

onde $\delta \mathrm{S}=\mathrm{S}_{\mathrm{f}}-\mathrm{S}_{\mathrm{s}}, \operatorname{com} \mathrm{S}_{\mathrm{f}} \mathrm{e} \mathrm{S}_{\mathrm{s}}$ representando as salinidades médias no fundo e na superfície, respectivamente, e $\mathrm{S}_{0}$ é a salinidade média na seçâo transversal. $V_{s}$ e $V_{f}$ são os valores médios da velocidade na superfície e a velocidade gerada pela descarga de água doce no sistema, respectivamente.

Com exceção de $V_{f}$, os valores dessas quantidades foram determinados experimentalmente com base nos valores horários medidos durante dois ciclos completos de maré e identificados numericamente com os seus valores médios. Para o cálculo de $\mathrm{V}_{\mathrm{f}}$ é necessário o conhecimento da descarga de água doce $\mathrm{Q}_{\mathrm{f}}$, pois $\mathrm{V}_{\mathrm{f}}=$ $\mathrm{Q}_{f} / \mathrm{A}$ (onde $\mathrm{A}$ é a área da seção transversal), cujo valor não é conhecido para o sistema em estudo. Assim, $V_{f}$ foi considerada, em primeira aproximação, igual ao valor da velocidade residual (média no tempo e ao longo da coluna de água), também calculada à partir dos valores médios horários obtidos em dois ciclos completos de maré. Modelos climáticos simplificados, tal como o apresentado em Kjerfve (1990), podem ser utilizados para estimativas de $\mathrm{Q}_{\mathrm{f}}$, mas o valor médio da velocidade gerada pela descarga de água doce calculada com esse valor não é adequado para o cálculo do parâmetro circulação.

\section{Resultados}

\section{Variaçäo local de propriedades: salinidade e velocidade}

A variação local da salinidade $(S)$ observada das $18,6 \mathrm{~h}$ do dia 05 às $09,0 \mathrm{~h}$ do dia 07 de dezembro de 1991 , é apresentada na Figura 2. Nesse período a estrutura da salinidade apresentou-se quase verticalmente

\section{Salinidade}

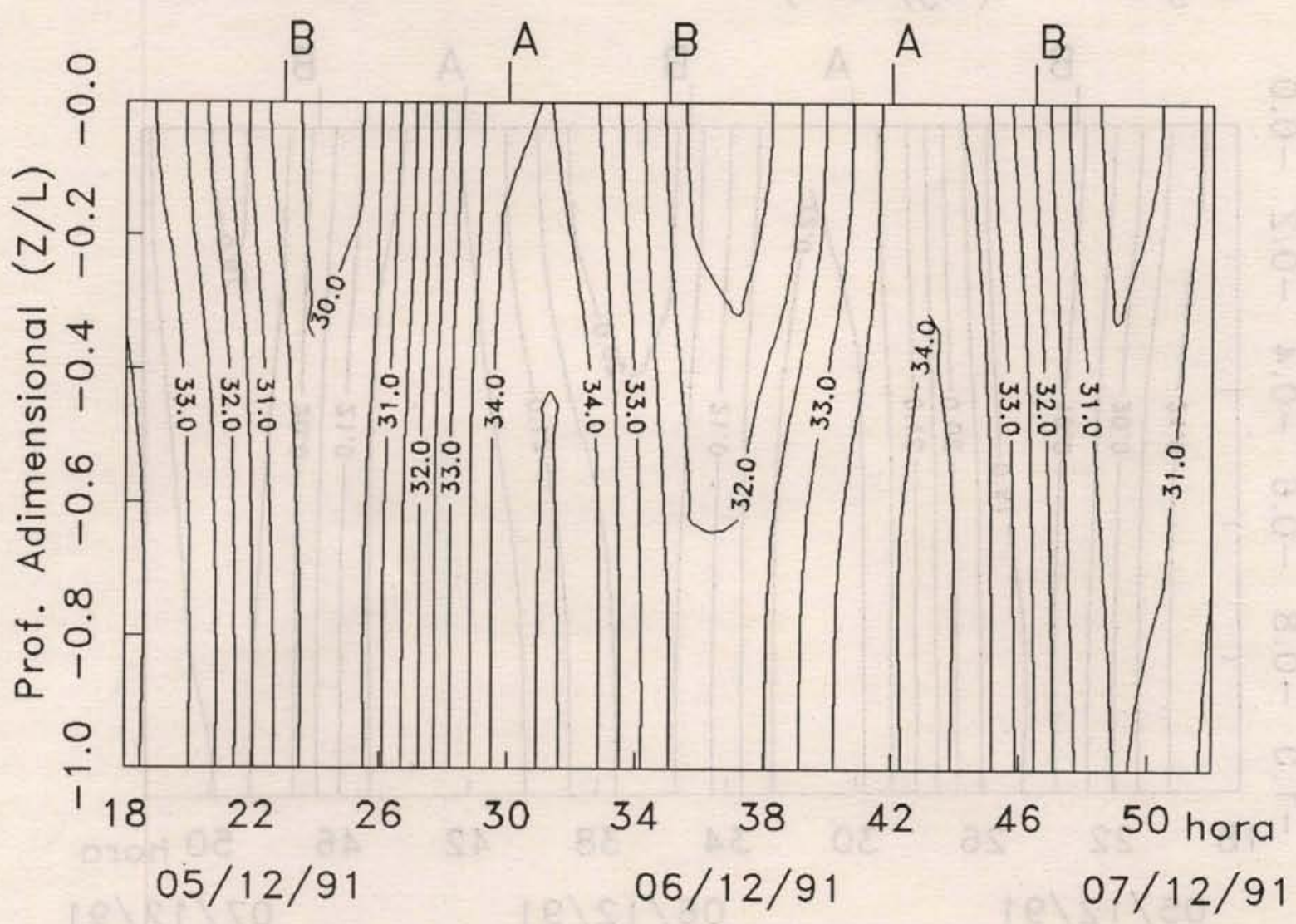

Fig. 2. Variabilidade local da salinidade, S, em \%o. A e B indicam os instantes de ocorrência das marés alta e baixa, respectivamente. Os intervalos entre as isohalinas são: $\Delta S=0,5 \%$. 
homogênea, notando-se apenas uma ligeira estratificação que ocorre nos instantes da reversão do sentido da corrente de maré. Enquanto essa propriedade variou no decorrer do tempo no intervalo $29<\mathrm{S}<35 \%$ o, a temperatura permaneceu quase constante, variando apenas entre 27 e $28^{\circ} \mathrm{C}$. A estrutura térmica apresentou uma ligeira inversão (aumento da temperatura com a profundidade), mas devido a sua pequena variabilidade, julgamos desnecessário a apresentação das isopletas dessa propriedade física, cuja influência sobre a estratificação de densidade é muito pequena.

Em decorrência da quase homogeneidade vertical da salinidade, os valores mínimos $(29,5-30,5 \%$ o ocorreram em núcleos na camada superficial. Esses extremos de salinidade estão associados a eventos de maré vazante, cerca de 2,0 a $3,0 \mathrm{~h}$ após a maré baixa (B), indicando a influência advectiva das águas de baixa salinidade do Mar Pequeno, que nesse estágio da maré foram transportadas em direção à estação fixa (Fig. 2). Os máximos valores da salinidade $(34,5-35,0 \%$ ) $)$ são observados em núcleos na camada superficial ou em profundidade, e os valores extremos desta propriedade estão associados a eventos de maré enchente (cerca de 1 a 2 h após a maré cheia - A), indicando a influência advectiva das águas mais salinas da região costeira adjacente.

A variabilidade das isopicnais $\left(\sigma_{t}\right)$ em função da profundidade e do tempo (Fig. 3) é muito semelhante à das isohalinas, ou seja, o campo de densidade é quase verticalmente homogêneo, apresentando apenas uma ligeira estratificação vertical que ocorre, tal como a da salinidade, nos instantes da reversâo do sentido da corrente de maré. A semelhança entre a configuração das isohalinas e das isopicnais mostra que o efeito da salinidade é preponderante sobre a densidade, característica esta comum aos sistemas estuarinos de planície costeira.

A variação local das componentes longitudinal (v) e transversal $(u)$ da velocidade da corrente são apresentadas nas Figuras $4 \mathrm{e} 5$, respectivamente. No início do período de observaçôes houve uma reversão no sentido do movimento de maré enchente $(v>0)$ para vazante $(v$ $<0)$, claramente identificada na estrutura vertical e temporal da componente $v$ (Fig. 4). Simultaneamente, observa-se um rápido decréscimo da salinidade e da densidade na coluna de água (Figs 2 e 3 ).

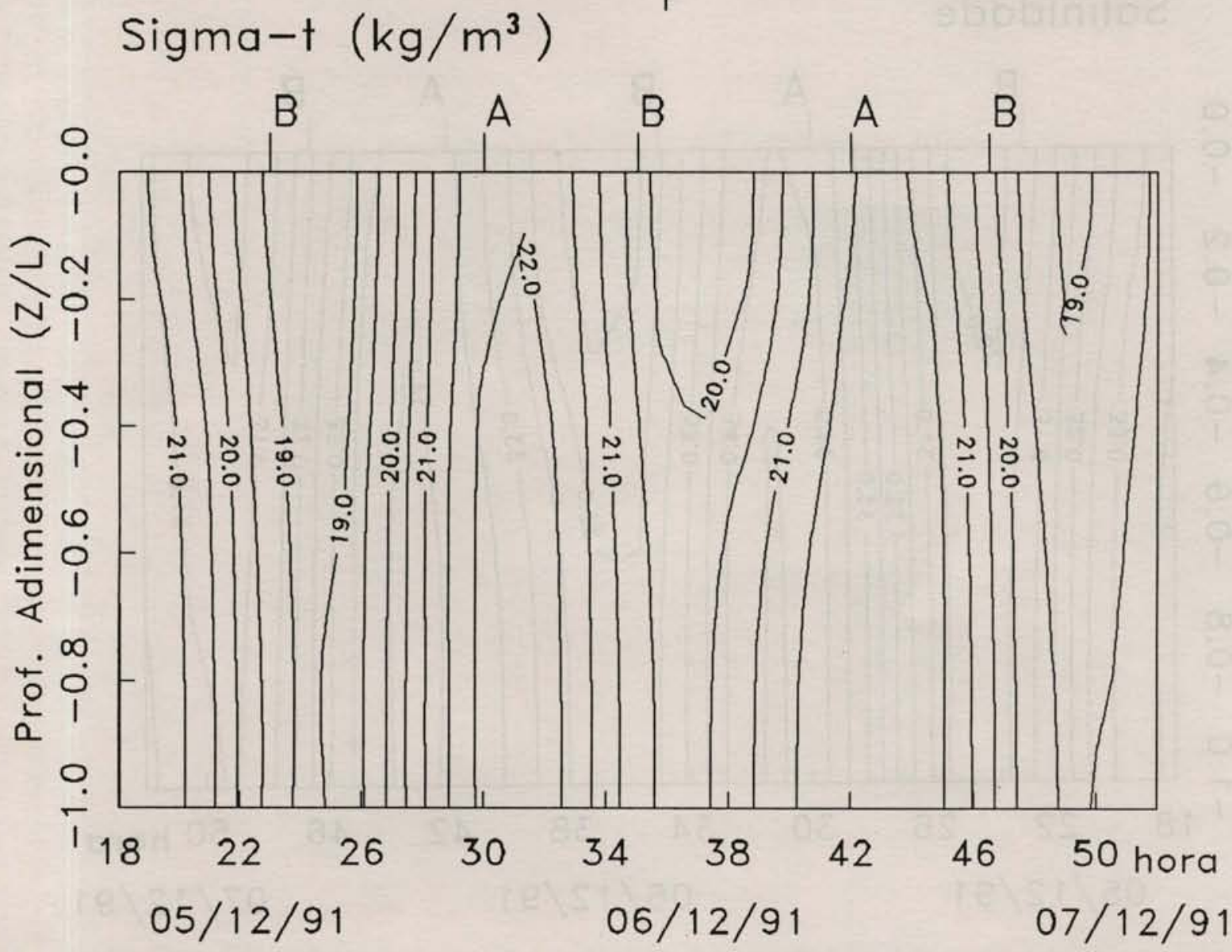

Fig. 3. Variabilidade local da anomalia da densidade à pressão atmosférica $\left(\sigma_{t}\right)$. A e B indicam os instantes de ocorrência das marés alta e baixa, respectivamente. Os intervalos entre as isopicnais são: $\Delta \sigma=0,5$. 
Componente $\vee(\mathrm{cm} / \mathrm{s})$

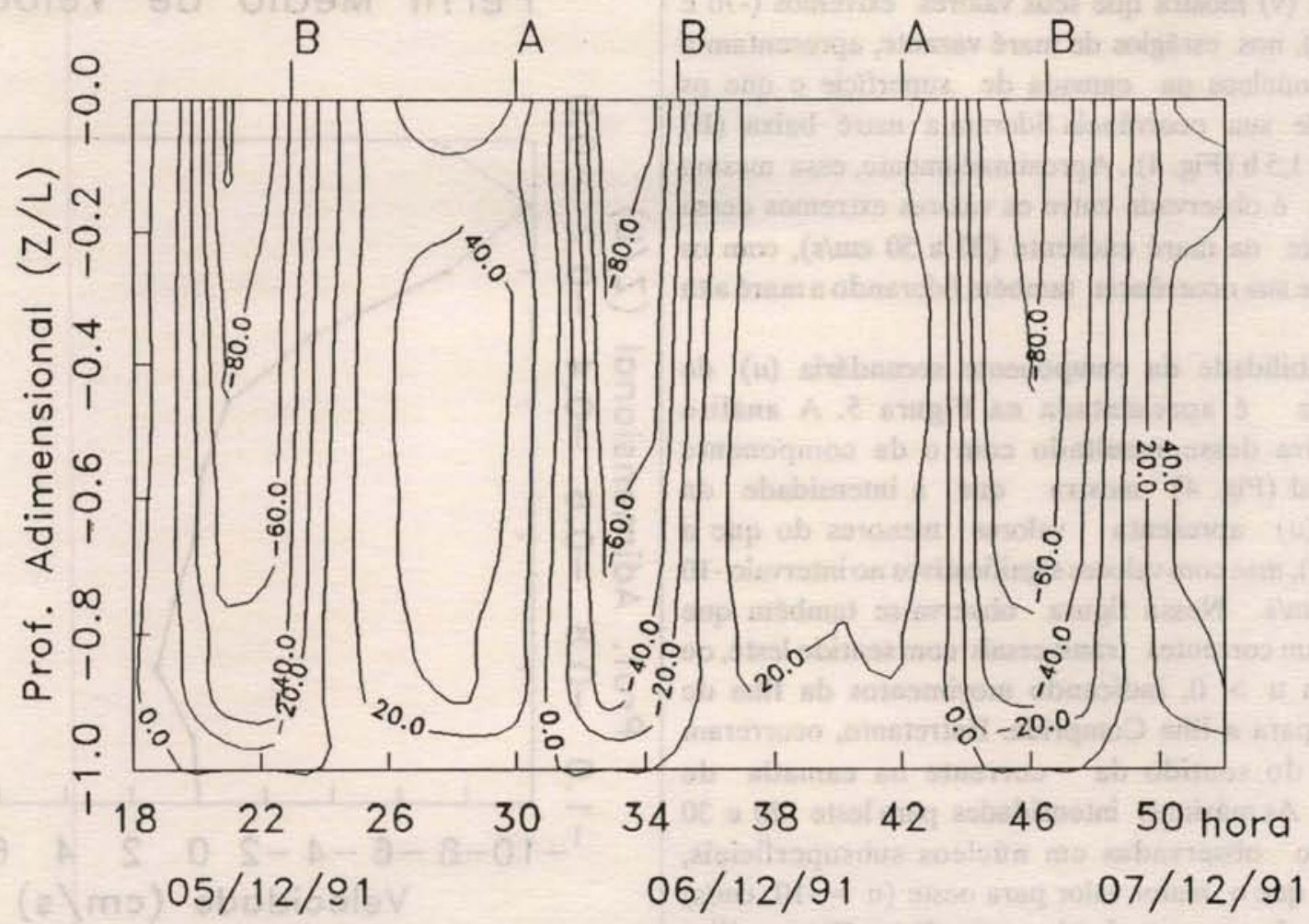

Fig. 4. Variabilidade local da componente longitudinal da velocidade da corrente, $v$. A e B indicam os instantes de ocorrência das marés alta e baixa, respectivamente. Os intervalos entre as isolinhas são: $\Delta v=20 \mathrm{~cm} / \mathrm{s}$.

\section{Componente $\mathrm{u}(\mathrm{cm} / \mathrm{s})$}

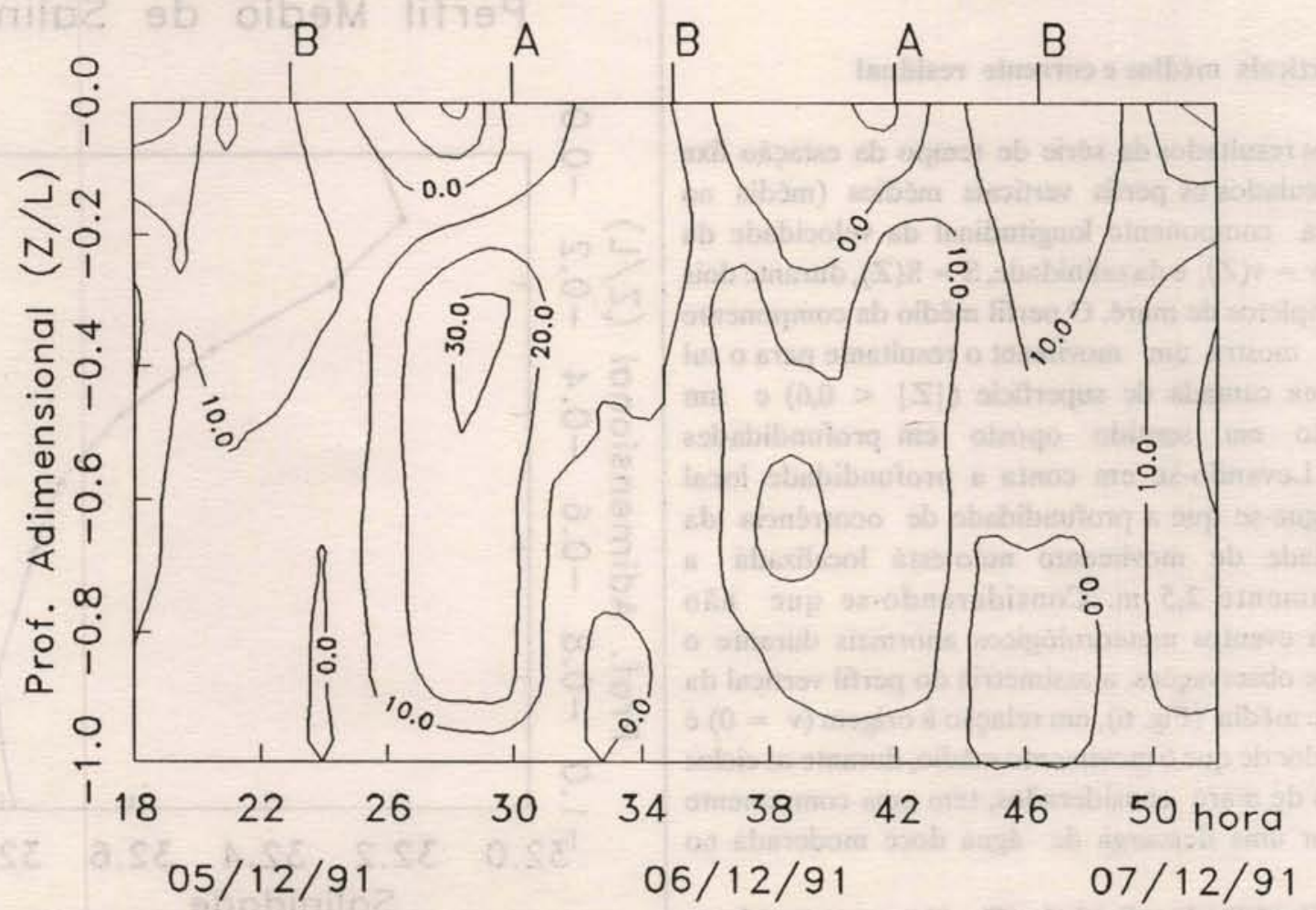

Fig. 5. Variabilidade local da componente transversal da velocidade da corrente, $u$. A e B indicam os instantes de ocorrência das marés alta e baixa, respectivamente. Os intervalos entre as isolinhas são: $\Delta v=10 \mathrm{~cm} / \mathrm{s}$. 
A variabilidade da componente longitudinal da velocidade (v) mostra que seus valores extremos $(-70 \mathrm{e}$ $-100 \mathrm{~cm} / \mathrm{s})$, nos estágios de maré vazante, apresentam a forma de núcleos na camada de superfície e que os instantes de sua ocorrência lideram a maré baixa (B) entre 1,0 a 1,5 h (Fig. 4). Aproximadamente, essa mesma defasagem é observada entre os valores extremos dessa componente na maré enchente ( 30 a $50 \mathrm{~cm} / \mathrm{s})$, com os instantes de sua ocorrência também liderando a maré alta (A).

A variabilidade da componente secundária $(u)$ de velocidade é apresentada na Figura 5. A análise comparativa desse resultado com o da componente longitudinal (Fig. 4) mostra que a intensidade da primeira $(u)$ apresenta valores menores do que a segunda (v), mas com valores significativos no intervalo - 10 e $30 \mathrm{~cm} / \mathrm{s}$. Nessa figura observa-se também que predominam correntes transversais com sentido leste, ou seja, com $\mathrm{u}>0$, indicando movimentos da Ilha de Cananéia para a Ilha Comprida. Entretanto, ocorreram inversōes do sentido da corrente na camada de superfície. As máximas intensidades para leste $(20$ e 30 $\mathrm{cm} / \mathrm{s}$ ) são observadas em núcleos subsuperficiais, enquanto que $o$ maior valor para oeste $(u=-10 \mathrm{~cm} / \mathrm{s})$ foi observado na camada de superfície. Essa análise comparativa também mostra que os extremos da componente secundária de velocidade estão associados ao instante de ocorrência dos máximos valores de velocidade na condição de maré enchente.

\section{Perfis verticais médios e corrente residual}

Comos resultados da série de tempo da estação fixa foram calculados os perfis verticais médios (média no tempo) da componente longitudinal da velocidade da corrente, $\mathrm{v}=\mathrm{v}(\mathrm{Z})$, e da salinidade, $\mathrm{S}=\mathrm{S}(\mathrm{Z})$, durante dois ciclos completos de maré. O perfil médio da componente $v$ (Fig. 6) mostra um moviment o resultante para o sul $(\mathrm{v}<0)$ na camada de superfície $(|\mathrm{Z}|<0,6)$ e um movimento em sentido oposto em profundidades maiores. Levando-se $\mathrm{cm}$ conta a profundidade local média, segue-se que a profundidade de ocorrência da profundidade de movimento nulo está localizada a aproximamente $2,5 \mathrm{~m}$. Considerando-se que não ocorreram eventos meteorológicos anormais durante $o$ período de observaçōes, a assimetria do perfil vertical da velocidade média (Fig. 6), em relação à origem $(v=0)$ é um indicador de que o movimento médio, durante os ciclos completos de maré considerados, tem uma componente gerada por uma descarga de água doce moderada no sistema.

O perfil médio da salinidade (Fig. 7) mostra uma fraca estratificação vertical, tal como evidenciado pelas

\section{Perfil Médio de Velocidade}

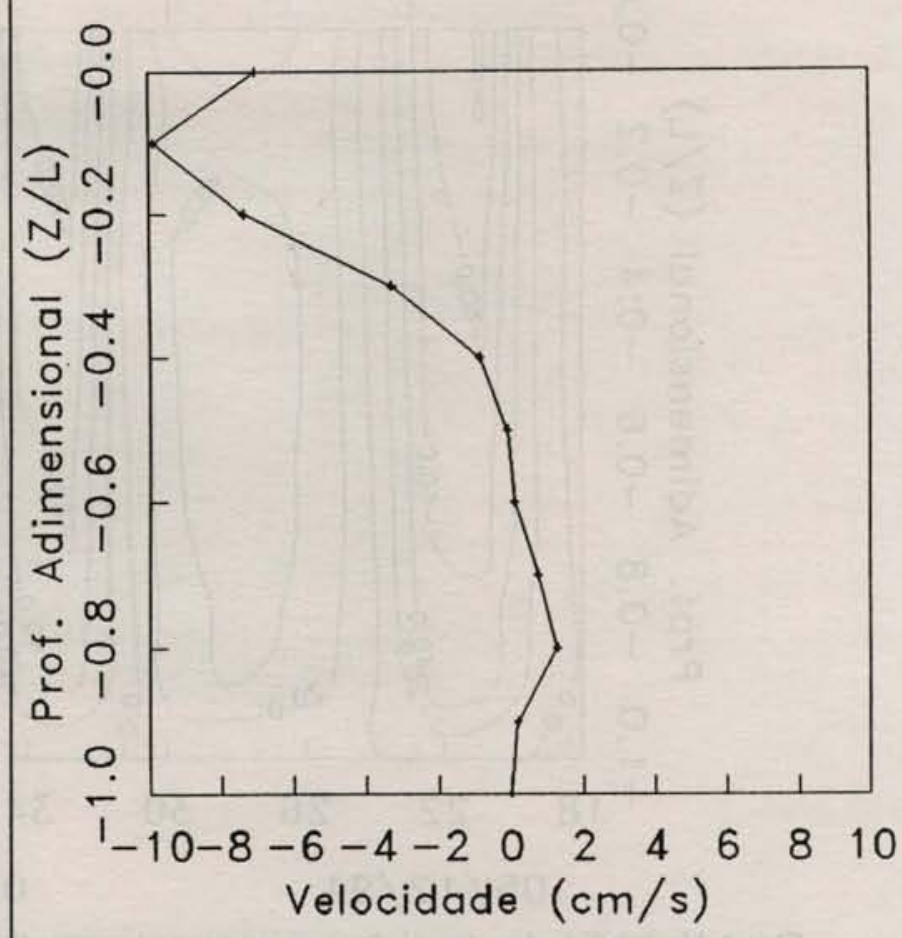

Fig. 6. Perfil vertical médio da componente longitudinal da velocidade da corrente, $v$, em $\mathrm{cm} / \mathrm{s}$.

\section{Perfil Médio de Salinidade}

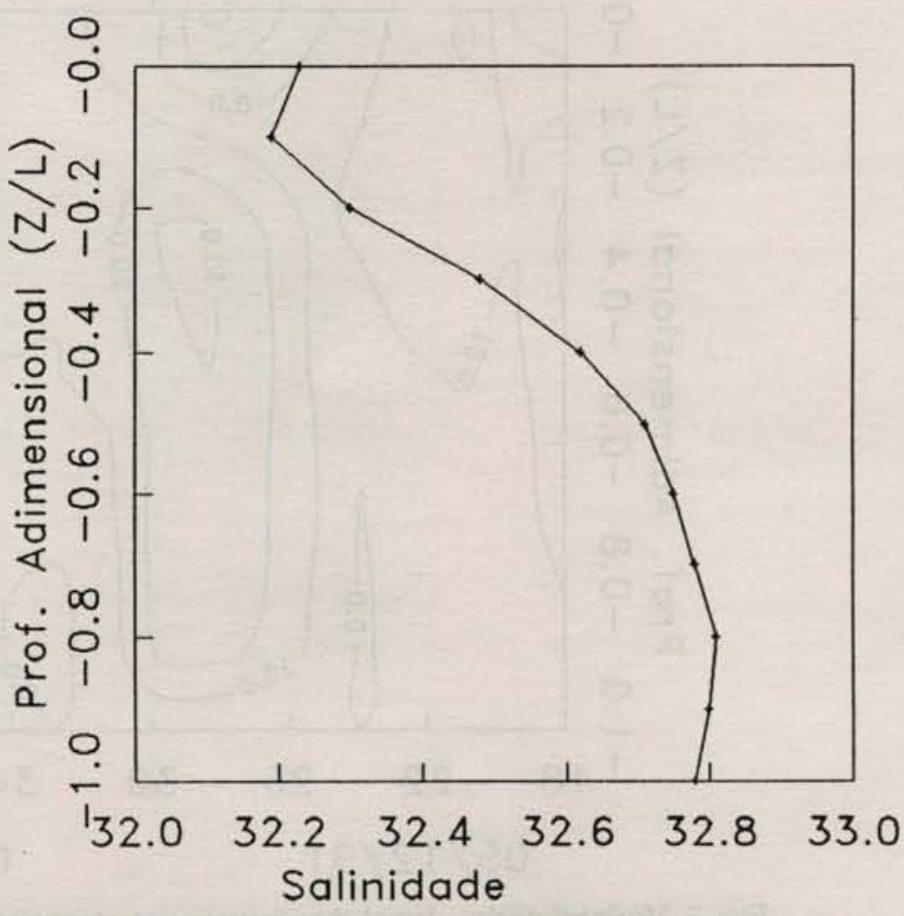

Fig. 7. Perfil vertical médio da salinidade, $\mathrm{S}$, em \%。. 
isopletas dessa propriedade (Fig. 2), cuja variação temporal foi analisada anteriormente. Utilizando esse perfil médio e o da componente longitudinal de velocidade (Fig. 6), foram também calculados os seguintes valores médios que serão necessários para determinar os parâmetros estratificação e circulação:

i - Valores médios (média no tempo) da velocidade na superfície $\left(V_{s}\right)$, e da salinidade na superfície $(S s)$ e no fundo $\left(\mathrm{S}_{\mathrm{f}}\right)$, respectivamente:

$$
\begin{aligned}
& \mathrm{V}_{\mathrm{s}}=-7,0 \mathrm{~cm} / \mathrm{s} \\
& \mathrm{S}_{\mathrm{s}}=32,2 \% 0 \\
& \mathrm{~S}_{\mathrm{f}}=32,8 \% 0
\end{aligned}
$$

ii - Valores médios da velocidade, $\langle\dot{\mathbf{v}}\rangle$ e da salinidade $(<\mathrm{S}>)$, ao longo da coluna de água:

$$
<\mathrm{V}>=-2,8 \mathrm{~cm} / \mathrm{se}<\mathrm{S}>=32,4 \% 0
$$

Considerando-se que a seção transversal que passa pela estação fixa (F) tem uma geometria relativamente simples pode-se assumir, em primeira aproximação, que as médias temporais e ao longo da coluna de água $(<\mathrm{V}>\mathrm{e}<\mathrm{S}>)$ são representativas para a velocidade residual e para o valor médio da salinidade nessa seçāo transversal; logo, esses valores médios podem ser identificados numericamente com a velocidade gerada pela descarga de água doce $\left(V_{f}\right)$ e com $\left(\mathrm{S}_{0}\right)$, respectivamente.

\section{Classificação da parte sul do Mar de Cananéia}

Com os dados obtidos na seçāo anterior foram calculados os seguintes valores para os parâmetros estratificação e circulação (eq. 3):

$$
\left(\delta \mathrm{S} / \mathrm{S}_{0}\right)=0,018 \text { e }\left(\mathrm{V}_{\mathrm{s}} / \mathrm{V}_{\mathrm{f}}\right)=2,5
$$

A correlação teórica entre os parâmetros estratificação e circulação é dada em função do parâmetro $v, o$ qual estabelece a proporção relativa dos processos advectivo e da difusāo turbulenta para ocasionar mistura. Essa correlação é apresentada na Figura 8, de acordo com Hansen \& Rattray (1966). A imagem dos parâmetros estratificaçāo e circulaçāo locada nessa figura é o ponto $\mathbf{P}$, indicando que o processo da difusāo turbulenta foi predominante para o fluxo de sal estuário acima $v \approx 1$.

Os valores dos parâmetros $\left(\delta \mathrm{S} / \mathrm{S}_{0}\right)$ e $\left(\mathrm{V}_{\mathrm{S}} / \mathrm{V}_{\mathrm{f}}\right)$ foram também locados no Diagrama Estratificação-circulação (Fig. 9), para a classificaçāo quantitativa do sistema estuarino investigado. $\mathrm{O}$ resultado obtido, também indicado pelo ponto $\mathbf{P}$, está localizado na parte do diagrama entre os trechos hachuriados, indicando que o sistema é parcialmente misturado e fracamente estratificado (Tipo 2a).

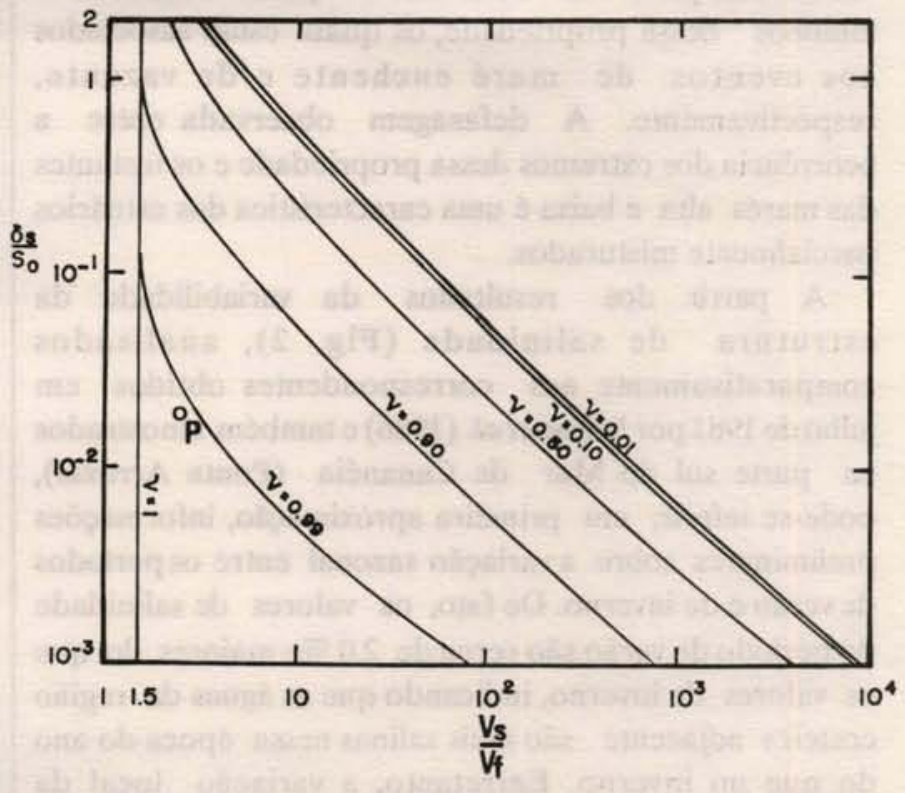

Fig. 8. Isolinhas da fração relativa $(v)$ do balanço horizontal de sal, gerada por difusão turbulenta, em função dos parâmetros estratificação e circulação, de acordo com Hansen e Rattray (1966). O ponto $P$ corresponde à imagem desses parâmetros obtidos para a parte sul do Mar de Cananéia.

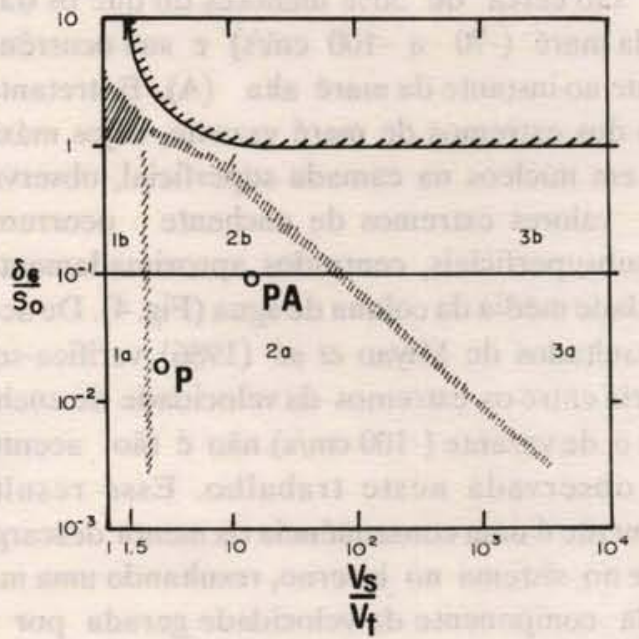

Fig. 9. Diagrama Estratificação-circulação, para a classificação de sistemas estuarinos, de acordo com Hansen e Rattray (1966). Os pontos P e PA são as imagens dos parâmetros estratificação $e$ circulação obtidos para a região em estudo e para a Baía de Paranaguá, respectivamente. 


\section{Discussão}

Pela configuração das isohalinas (Fig. 2), verifica-se que a influência advectiva das águas mais salinas da região costeira adjacente e das águas de origem continental pode ser identificada pelos máximos e mínimos dessa propriedade, os quais estão associados aos eventos de maré enchente e de vazante, respectivamente. A defasagem observada entre a ocorrência dos extremos dessa propriedade e os instantes das marés alta e baixa é uma característica dos estuários parcialmente misturados.

A partir dos resultados da variabilidade da estrutura de salinidade (Fig. 2), analisados comparativamente aos correspondentes obtidos em julho de 1981 por Miyao et al. (1986) e também amostrados na parte sul do Mar de Cananéia (Ponta Arrozal), pode-se inferir, em primeira aproximação, informações preliminares sobre a variação sazonal entre os períodos de verão e de inverno. De fato, os valores de salinidade do período de verão são cerca de $2,0 \%$ maiores do que os valores de inverno, indicando que as águas da região costeira adjacente são mais salinas nessa época do ano do que no inverno. Entretanto, a variação local da salinidade apresenta característica sazonal semelhante, ou seja, a estrutura de salinidade apresenta-se verticalmente homogênea em torno das correntes máximas de enchente e de vazante, com uma ligeira estratificaçāo nas estofas. A mesma característica pode ser observada na variabilidade de $\sigma_{\mathrm{t}}$ (Fig. 3).

Os valores máximos da intensidade da componente longitudinal da corrente (v) na maré enchente (entre $30 \mathrm{e}$ $50 \mathrm{~cm} / \mathrm{s}$ ) são cerca de $50 \%$ menores do que os da fase vazante da maré $(-70$ a $-100 \mathrm{~cm} / \mathrm{s})$ e sua ocorrência é precedente ao instante da maré alta (A). Entretanto, ao contrário dos extremos de maré vazante, cujos máximos ocorrem em núcleos na camada superficial, observa-se que os valores extremos de enchente ocorrem em núcleos subsuperficiais, centrados aproximadamente na profundidade média da coluna de água (Fig. 4). De acordo com os resultados de Miyao et al. (1986), verifica-se que a assimetria entre os extremos da velocidade de enchente $(50 \mathrm{~cm} / \mathrm{s})$ e de vazante $(-100 \mathrm{~cm} / \mathrm{s})$ não é tão acentuada como a observada neste trabalho. Esse resultado provavelmentc ć uma conseqüência da menor descarga de água doce no sistema no inverno, resultando uma menor parcela da componente da velocidade gerada por essa descarga.

O exame da Figura 4 também evidencia a assimetria entre os extremos da velocidade de enchente e de vazante, notando-se também que os intervalos de tempo de enchente são maiores que os de vazante. Essc resultado confirma os obtidos por Miyao \& Harari (1989), com base na análise de uma séric de tempo da velocidade da corrente a $2 \mathrm{~m}$ do fundo, registrada num fundeio localizado $8 \mathrm{~km}$ ao norte da estação fixa $\mathbf{F}$ (Fig. 1).

Foram também observadas defasagens entre os extremos de velocidade e os instantes de ocorrência das marés alta (A) e baixa (B). Esse fato, associado ao intenso cisalhamento vertical da velocidade, sugere a ação dos efeitos associados das componentes barotrópica e baroclínica da força de gradiente de pressão e a influência dissipativa da força de atrito com o fundo do canal.

O perfil médio de velocidade (Fig. 6) é característico de sistemas estuarinos de planície costeira fracamente estratificados. A forçante responsável pela ocorrência dessa estrutura média de velocidade é a ação das componentes da força de gradiente de pressão, associada à descarga de água doce no sistema. Uma descrição detalhada da influência dessas componentes sobre a estrutura vertical de velocidade média, sob diferentes condiçōes da descarga de água doce e nas fases de maré enchente e vazante no Mar de Cananéia, é apresentada em Miranda (1990).

O perfil médio de salinidade (Fig. 6) permite, de acordo com o critério da estratificação vertical da salinidade (Pritchard, 1952; Cameron \& Pritchard, 1963; Officer, 1977), classificar o sistema estuarino investigado como do tipo fracamente estratificado.

Como a parte do sistema estuarino em estudo tem uma geometria simples, pode-se assumir por hipótese que os parâmetros estratificação e circulação calculados são representativos para a seção transversal. Assim, com a utilização do Diagrama Estratificação- circulação (Fig. 9), idealizado por Hansen \& Rattray (1966), fica demonstrado que para o transporte de sal estuário acima, o processo de difusão turbulenta teve uma influência predominante, enquanto que a contribuiçāo do processo advectivo foi quase desprezível. De acordo com esse diagrama, o trecho do mar de Cananéia estudado foi classificado como parcialmente misturado e fracamente estratificado (Tipo 2a). Para fins de comparação, locou-se também nesse diagrama os valores desses parâmetros determinados, em primeira aproximação, por Knoppers et al. (1987) para a Baía de Paranaguá que interliga-se ao sul com a regiāo pesquisada. $\mathrm{A}$ imagem desses parâmetros sobre esse diagrama (PA) indica que na época dos experimentos o trecho investigado dessa baía classifica-se na transição entre os Tipos $\mathbf{2 a}-\mathbf{2 b}$, apresentando também a predominância do processo de difusão turbulenta para o transporte de sal.

\section{Conclusões}

A variação temporal das isohalinas de salinidade apresenta uma fraca estratificação vertical. Os máximos e mínimos dessa propriedade estão defasados dos 
instantes de ocorrência das marés alta e baixa, respectivamente, e seus valores extremos indicam a influência das águas mais salinas da região costeira adjacente e da descarga de água doce no sistema estuarino.

As defasagens observadas entre os instantes de ocorrência das maré alta e baixa e os extremos da componente longitudinal da velocidade da corrente e da salinidade indicam que a propagação da maré é uma combinação de ondas progressiva e estacionária, característica de sistemas estuarinos parcialmente estratificados, confirmando resultados de pesquisas anteriores realizadas no Mar de Cananéia.

Os extremos da componente transversal de velocidade $(-10$ e $30 \mathrm{~cm} / \mathrm{s})$ foram observados quando da ocorrência dos extremos da componente longitudinal durante a maré enchente $(50 \mathrm{~cm} / \mathrm{s})$.

A assimetria e a reversão da velocidade observada no perfil médio indicam que a circulação é principalmente forçada pela maré, desempenhando a componente baroclínica da força de gradiente de pressão um efeito secundário.

Embora apresentando características de um sistema do tipo construído por barras, de acordo com a sua geomorfologia, a regiāo em estudo não se enquadra na definição clássica de um estuário. Entretanto, a aplicação do Diagrama Estratificação-circulação mostrou que o extremo sul do Mar de Cananéia na época do experimento era do tipo parcialmente misturado e fracamente estratificado (Tipo 2a) e o balanço de sal, ocasionando a mistura da água do mar com a água doce, foi predominantemente gerado por difusão turbulenta.

\section{Agradecimentos}

Aos técnicos do Laboratório de Instrumentação Oceanográfica (LIO) do Departamento de Oceanografia Física do Instituto Oceanográfico da USP, aos tripulantes do Barco de Pesquisa Albacora e a Gilberto Ivo Sarti pela arte final de desenhos. Este trabalho foi possível graças ao auxílio financeiro da FAPESP (Proc. 87/0206-1) dado ao segundo autor para a realização dos experimentos. Nossos agradecimentos também ao CNPq pela Bolsa de Produtividade em Pesquisa concedida ao primeiro autor (Proc. 301014-79-5).

\section{Referências bibliográficas}

BESNARD, W. 1950a. Considerações gerais em torno da regiāo lagunar de Cananéia-Iguape. I. Bolm Inst. paul. Oceanogr., 1(1):9-26.
BESNARD, W. 1950b. Consideraçōes gerais em torno da região lagunar de Cananéia-Iguape. II. Bolm Inst. paul. Oceanogr., 1(2):3-28.

CAMERON, W. M. \& PRITCHARD, D. W. 1963. Estuaries. In: Hill, M. N. The sea. New York, John Willey. 2:306-324.

KNOPPERS, B. A.; BRANDINI, F. P. \& THAMM, C. A. 1987. Ecological studies in the Bay of Paranaguá. II. Some Physical and Chemical characteristics. Nerítica, Pontal do Sul, 2(1):1-36.

ICES. International Council for the Exploration of the Sea. 1966. Oceanographic punch cards. $2 \mathrm{nd}$ ed. Copenhague, $15 \mathrm{p}$.

HANSEN, D. V. \& RATTRAY, M. 1966. New dimensions in estuarine classification. Limnol. Oceanogr., 11:319-326.

JOHANNESSEN, O. M. 1967. Preliminary studies on the mean daily oscilations of sea level and atmospheric pressure at Cananéia and Santos on the southern part of the Brazilian coast. Contrçōes Inst. oceanogr. Univ. S Paulo, sér. Oceanogr. fís., (9):8-15.

KJERFVE, B. 1975. Velocity averaging in estuaries characterized by a large tidal range to depth ratio. Estuar. coast. mar. Sci., (3):311-323.

1990. Manual for investigation of hydrological processes in mangrove ecosystems. UNESCO/UNDP, New Delhi, 79 p.

LORENZZETTI, J. A. 1976. Aplicação da técnica de análise espectral cruzada ao estudo da correlação entre as oscilações do nível do mar observadas em Cananéia e Bom Abrigo. Dissertação de mestrado. Universidade de São Paulo, Instituto Oceanográfico. $118 \mathrm{p}$.

MACHADO, L. B. 1952. Pesquisas físicas e químicas do sistema hidrográfico da região lagunar de Cananéia II. Águas de junção. Estudos iniciais das águas comuns da região Ilha da Casca. Nota preliminar sobre as águas na junção do Canal de Ararapira e Baia de Trapandé. Bolm Inst. oceanogr., S Paulo, 3(1/2):55-75.

MESQUITA, A. R. DE \& HARARI, J. 1983. Tides and tide gauges of Cananéia and Ubatuba - Brazil (lat. $24^{\circ}$ ). Relat. int. Inst. oceanogr. Univ. S Paulo, (11):1-14. 
MESQUITA, A. R. de \& HARARI, J. 1993. Tábuas de marés de Ubatuba e Cananéia para os anos de 1994 e 1995. Relat. int. Inst. oceanogr. Univ. S Paulo, (35):1-20.

MINIUSSI, I. C. 1959. Propagação da maré em torno da Ilha de Cananéia. Contrçōes Inst. oceanogr. Univ. S. Paulo, sér., Oceanogr. fís,. (2):1-5.

MIRANDA, L. B. de 1984. Cinemática e dinâmica de estuários. São Paulo, BSP. 360 p.

1990. Sistemas estuarinos de planície costeira: estrutura dinâmica, processos de mistura e aplicações. In: SIMPÓSIO DE ECOSSITEMAS DA COSTA SUL E SUDESTE BRASILEIRA: ESTRUTURA, FUNÇĀO E MANEJO. 2. Águas de Lindóia, 1990. ACIESP, São Paulo, 71(2):1-46.

MIYAO, S. Y. 1977. Contribuição ao estudo da Oceanografia Física da região de Cananéia (lat. $25^{\circ} \mathrm{S}$ long. $048^{\circ} \mathrm{W}$ ). Dissertação de mestrado. Universidade de São Paulo, Instituto Oceanográfico. 87 p.

; NISHIHARA, L. \& SARTI, C. C. 1986. Características Físicas e Químicas do Sistema Estuarino- Lagunar de Cananéia-Iguape. Bolm Inst. oceanogr., S Paulo, 34(único):23-36.

\& HARARI, J. 1989. Estudo preliminar da maré e das correntes de maré na regiāo estuarina de Cananéia $\left(25^{\circ} \mathrm{S}-048^{\circ} \mathrm{W}\right)$. Bolm Inst. oceanogr., S Paulo, 37(2):107-123.
OCCHIPINTI, A. G. 1963. Climatologia dinâmica do litoral sul brasileiro. Contrçōes Inst. oceanogr. Univ. S Paulo, sér. Oceanogr. fís., (3):1-86.

OFFICER, C. B. 1977. Longitudinal circulation and mixing relations in estuaries. In: Estuaries, Geophysics and the Enrironment. National Academy of Sciences, Washington, D.C. p. 13-21.

PRITCHARD, D. W. 1952. Salinity distribution and circulation in the Chesapeake Bay estuarine system. J. mar. Res., 11(1):106-123.

SILVA, J. F. da 1989. Dados climatológicos de Cananéia e Ubatuba (Estado de São Paulo). Bolm climatol. Inst. oceanogr., S Paulo, (6):1-21.

TUNDISI, J. G. 1969. Produção primária, "standing-stock" e fracionamento do fitoplâncton na região lagunar de Cananéia. Tese de doutorado, Universidade de São Paulo, FFLCH.130 p.

(Manuscrito recebido 13 jullho 1994; aceito 09 novembro de 1995) 